\title{
Formal Verification of Autonomous UAV Behavior for Inspection Tasks Using the Knowledge Base System IDP
}

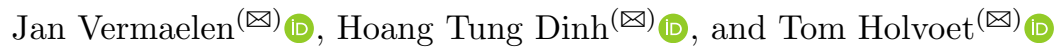 \\ Department of Computer Science, imec-DistriNet, KU Leuven, \\ 3001 Leuven, Belgium \\ $\{$ jan.vermaelen, hoangtung.dinh, tom.holvoet $\}$ @cs. kuleuven. be
}

\begin{abstract}
Unmanned Aerial Vehicles (UAVs) have become useful tools in industries. In this paper, we verify the behavior of an autonomous UAV executing an inspection task. More specifically, we look into the use of the knowledge base system IDP as a verification tool. We propose an approach for the modeling and verification of the safety-critical UAV and its environment in IDP. The methodology and modeling choices that are beneficial for the performance of the verification task and the readability of the model are denoted. We identify the need for discrete domains and investigate the consequences. Verification is successfully achieved using both Bounded Model Checking (BMC) and Invariant Checking (IC).
\end{abstract}

Keywords: IDP - Unmanned Aerial Vehicle • Autonomous system • Model $\cdot$ Verification

\section{Introduction}

In recent years, Unmanned Aerial Vehicles (UAVs) have become increasingly popular in industries. They are, compared to human performance, very fast and agile. The interest arose to use them for various tasks, such as inspection tasks. The UAV can be equipped with an autopilot to automate the process. Companies providing inspection and monitoring services using UAV systems already exist.

The application verified in this research originates from a setting in which a UAV operates on an industrial site, inspecting the state, integrity, and operation of machines, reservoirs, and buildings. The flight goal of the UAV consists of autonomously achieving a visual inspection of artifacts at several predefined locations. Achieving this goal requires the execution of autonomous actions regarding take-off, navigation, height adjustment, capturing images, and landing, as well as the correct scheduling of these actions.

The UAV might be deployed along with human actors and potentially hazardous equipment. Since the UAV is a safety-critical system, safety and productivity properties of its behavior are important. It is interesting, if not essential, to be able to verify these properties to guarantee them to a certain extent. From 
a safety point of view, one wants to be able to make sure that things that should never happen, indeed do not happen. A straightforward, yet highly relevant example states that the UAV should never fly into other agents or equipment. On the other hand, things one does want to happen, should eventually happen, to make sure the pilot gets work done. The UAV should make progress towards its overall flight goal. This distinction will result in the verification of safety properties and liveness properties, respectively.

For the verification task, we look into the use of IDP $^{1}$, a knowledge base system. The modeling language, $\mathrm{FO}($.$) , is an extension of traditional First Order$ logic [5]. It allows for modeling using (inductive) definitions and provides a type system with some built-in types and support for user-defined types. IDP only supports types with discrete domains. This limitation confines the sorts of systems that can be modeled, yet for the application at hand, the consequences should not be too severe. It follows that the result of the verification task is only valid under the assumption that the application can be (and is) modeled correctly in this discrete setting. Furthermore, when using a knowledge base system, the implementation of a concrete verification approach is up to the user. We verify properties using Bounded Model Checking (BMC) and Invariant Checking (IC).

The overall contribution of this work consists of an IDP model of the UAV and its environment. We focus on the advantages and disadvantages of using IDP for verification purposes. Preliminary examples show that IDP can be used, yet only for relatively small systems. How can IDP be used as a model checking tool for the application at hand? What is the desired approach? To what level of detail can the system be modeled? What precautions can be undertaken to maintain tractability? We look into the modeling aspect of the application and denote the preferred modeling approaches. To model the system precisely, we keep the state description as concrete as possible. The need emerges to use numerical modeling instead of a higher-level description. When relevant, subtypes are introduced to improve the performance and readability of the model. Non-determinism is addressed to model more realistic, uncertain effects. Finally, the concept of a back loop time path (instead of a linear, finite trace) provides support for stronger guarantees.

The paper is organized as follows. Section 2 discusses other research verifying the behavior of UAVs. Section 3 elaborates on the outline of our model and choices that were made during modeling. Section 4 discusses the results and findings. Section 5 draws the conclusions and outlines future work.

\section{Related Work}

As mentioned above, it is important to be aware of the safety and productivity properties of a safety-critical system and to be able to verify and guarantee such properties. A lot of research has been going on regarding model checking and verification. In our work, we do not introduce a novel or superior model checking

\footnotetext{
${ }^{1}$ IDP stands for Imperative Declarative Programming. More information on IDP and its modeling language can be found here: https://dtai.cs.kuleuven.be/software/idp.
} 
tool or technique. We are merely interested in taking a knowledge base approach for verification purposes, more specifically, using the IDP system. Despite IDP being able to perform verification tasks, little research has been investigating this ability [7]. In this section, we touch upon some verification approaches and applications related to UAV systems. From the point of view of model checking within IDP, the verification of other systems is considered relevant as well.

The techniques we will apply within IDP are majorly based on BMC principles. Biere et al. [3] provide a solid basis regarding a BMC approach. The idea emerges to search for counterexamples of a property to reject this property. If an execution trace of a bounded length is found, in which the property gets violated, the property does not hold. If no counterexample is found, the bound has to be increased until one is found, or until some pre-known upper bound is reached. The property holds if no counterexample can be found.

Torens et al. [12] emphasize the need for verification of UAV software and behavior. Many regulatory rules and certification standards have to be taken into account during the development of the autopilot. This leads to numerous challenges. A NASA case study [10] looks into autonomy software and the issues related to verification. Interesting to note is that behavioral errors are considered more difficult to detect, compared to, for example, implementation errors. The used models form a critical basis. Sirigineedi et al. [11] look into the modeling and verification of a multiple UAV system. The properties are verified using counterexamples. Unlike their approach, our work concerns the properties of the behavior of a single UAV. This approach allows us to focus on the modeling details and choices of the UAV itself. Alhawi et al. [1] investigate the verification of UAV systems, to detect security vulnerabilities. Their work takes into account the communication aspect of the system. We consider the autopilot to reside onboard the UAV. No external communication is required, avoiding another opportunity for failure or attack.

Finally, as elaborated upon by Hoffmann et al. [8], the use of probabilistic model checking can yield more informative results. For this purpose, the probabilistic model checker PRISM [9] is used. Actions can have probabilistic effects, yielding more realistic models, compared to models in IDP. Furthermore, probabilistic guarantees (expressing chances) can be obtained, rather than less informative boolean results (expressing possibilities). However, a sufficiently precise probabilistic model of the effects of actions on the UAV and world is required. Such a precise model can be hard to obtain.

\section{Modeling the System}

A typical inspection flight roughly looks as follows. The UAV starts at its home base location, landed on the ground, where it is deployed or serviced. When a mission is due, the UAV will take off and rise to an appropriate flight height. At this height, where obstacles are least common and no legal restrictions hold, the most direct path to the inspection location is available. The autopilot will navigate the UAV to the inspection location, actively avoiding (possibly temporal and dynamic) restricted regions representing other UAVs, tall buildings, 
and restricted airspace. At the inspection location, the UAV will lower to an appropriate inspection height. At this position, inspection is due. Currently, this inspection corresponds with taking a picture of which the processing will be handled offline. After the inspection, the UAV can regain height and travel onward to the next location. This location can be the next inspection location or the home base if no more inspections are due. Furthermore, the UAV is not allowed to enter a height zone above a certain height level, defined by law.

\subsection{Modeling in IDP}

Before we can verify the properties of a system, the system has to be modeled in IDP. The modeling language $\mathrm{FO}($.$) allows for straightforward modeling of the$ system and its properties. In IDP, coherent knowledge, expressing (a part of) the model is grouped in a theory. The base theories contain the autopilot policy ${ }^{2}$ and the UAV and its environment. Furthermore, time is modeled explicitly to support a BMC approach. The obtained model allows us to use IDP to search for execution traces of the UAV that meet certain constraints. We will use this approach to find counterexamples of properties, to reject these properties.

A concrete, numerical representation of the state of the system is deemed to be more informative than a boolean indicative approach. For example, an integer percentage as a representation for the battery level of the UAV can represent the complete battery range. A boolean indication, battery_below_n_\%, only expresses whether or not a threshold is reached. It does not make sense to discard the fine-grained information present in real systems. This information might be required to model the correct physical behavior. For example, since the battery drains slowly during each action, the UAV will fall to the ground once all power is drained. The disadvantage of keeping track of the detailed information is the need for larger domains, for example, $\{0,1, \ldots, 100\}(\%)$ instead of $\{0,1\}$ (boolean). Large domains are costly in terms of performance. The relevant domains are (manually) modeled as small as possible, while still providing the necessary features.

It has to be said that an integer representation is the most concrete type available in IDP. A more exact, continuous representation might be preferred, but this is not supported. We use discretized values, giving rise to a less precise model. The verification capabilities for the model at hand should not be affected. In general, the precise effects of the actions of a system can not always be modeled using a discrete domain. For some actions, the possible effects might not even be considered to be accurately known. Under the assumption that the actions of the system have accurately known effects, a discretized approach yields a valid model. Conceptually, to achieve a more fine-grained representation, one can increase the size of the relevant domains. For example, represent the distance using centimeters instead of meters. In our model, we generalize over the specific units being used.

\footnotetext{
${ }^{2}$ A policy contains a mapping from every possible state of the system to the action that has to be executed in that state.
} 


\subsection{Modeling the UAV and World}

The complete model ${ }^{3}$ makes use of many (helper) predicates and (helper) functions. The most significant types for defining the (physical) state of the UAV are the following.

Location is a type used to declare the meaning of the different height independent locations at which the UAV can be. This approach is chosen, rather than a 3D discretized world grid, as the latter yields to be intractable in IDP. Without the usage of $3 \mathrm{D}$ coordinates in the model, we are not able to represent every possible location. We solely define the world using the distances between the locations. This approach provides all the information needed to model any flight between the locations. Location is constructed from the values:

- Home being the home base of the UAV.

- TravelSpace being the less interesting part of the world through which the UAV can fly to reach the other locations.

- A number of inspection locations Insp $_{\mathbf{n}}$ at which inspection is due.

An example toy world is shown in Fig. 1. The distances between different locations are fixed in time so that the locations in the world are static. As the UAV flies around in the world, the distances between the UAV and the locations will change deterministically. When the UAV is navigating from location $A$ to location $B$, the distance between the UAV and location $A$ increases. The distance between the UAV and location $B$ decreases. The distances between the UAV and other locations remain constant. This approach is solely a (physically incorrect) modeling choice and is implemented so that it does not compromise the correctness of the verification task. A physically correct model could be preferred but would require continuous domains and calculations.

The restricted area, which is to be avoided by the UAV, is modeled less explicitly. The distance between the UAV and RestrictedArea can change nondeterministically ${ }^{4}$ during flight. As a result, the restricted area has no fixed shape. IDP can verify properties using the most diverse and dynamic shapes possible, which can represent (the behavior of) humans and other agents.

Height is a natural number, used to represent the current height of the UAV. As an example illustrates in Fig. 2, Height has the following distinct subtypes:

- GroundHeight representing the landed position.

- InspectionHeight being the lower level of the air space, in which objects are to be inspected.

3 The IDP model of the application at hand is available at https://github.com/ VermaelenJan/Model-Checking-Drones-with-IDP/blob/master/model.idp.

${ }^{4}$ Non-determinism in IDP can be achieved by defining a predicate without initializing it. IDP will look for models regarding all possible values (of the relevant domain) for the predicate. 
- FlyHeight being the higher level of the air space, in which the UAV is more or less free to fly in.

- RestrictedHeight being the highest level of the air space, in which the UAV is not allowed to fly, for legal reasons.

This height representation outlines the complete physical air space. Every concrete altitude in the real aerial space is (non-linearly) mapped to one numerical height value and thus to one of these levels. This representation is interesting, both for the readability of the model and for the performance of the verification task. Certain instructions or statements are only relevant for (the domain of) one such region. For example, inspection is due only at InspectionHeight. As the computations within IDP can be executed using smaller domains, a performance gain (linear with the reduction) is observed.

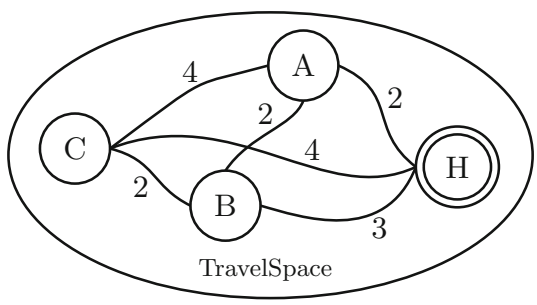

Fig. 1. The locations (Home base $\mathrm{H}$, Inspection Locations A, B and C, and the TravelSpace in between) of a simple toy world are visualized, along with the distances connecting them.

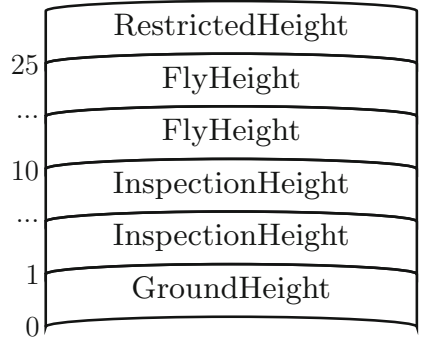

Fig. 2. The height definition is visualized, showing the semantic height zones and the boundaries between them as they are present in a simple toy world.

Power is a natural number, used to represent the quantity of power. This numerical value is, for example, used by helper predicates to determine whether the battery of the UAV is at time step t:

- AtNormalPower(t) when normal operation of the UAV is expected.

- AtLowPower(t) when the UAV is expected to return home.

- AtCriticalPower(t) when the UAV is expected to land immediately.

The numerical value keeps track of the actual power level of the battery. Once the value reaches zero, the UAV can no longer execute any actions since each action requires power to execute. The UAV will start falling to the ground. The effect of this falling is modeled as an unconditional lost of altitude at each consecutive time step.

An intuitive domain to model the PowerUsage of the UAV, that is, the power used at each time step, would be the same Power domain that is being used for 
the battery itself. However, the values for PowerUsage are typically much smaller than an entire battery worth of power. This makes PowerUsage suitable to be modeled as a subtype of Power. Analogous to the modeling of the Height, the use of a subdomain improves tractability and the readability of the model.

Action is a type used to represent the possible actions that can be executed by the UAV on behalf of the autopilot. The policy (see Sect. 3.3) will be responsible for deciding which actions to execute, based on the state of the system. The possible actions are:

- MoveTowardsTarget to (try to) move towards the current Target location.

- MoveAwayFromRA to (try to) move away from the closest region modeled as RestrictedArea.

- TakePicture to take a picture at the current position.

- NoOp to execute no operation. This action will, for example, occur after the UAV has completed its tasks.

- Lift to (try to) gain altitude.

- Lower to descend.

The Move and Lift actions are modeled so that they can fail a (nondeterministic) number of times. Assumptions on the maximum amount of successive or total failures can be set as a function of the weight. A heavy UAV could take longer to lift or move. Furthermore, each action is modeled to have a certain power consumption, gradually draining the battery.

\subsection{Modeling the UAV Behavior}

As mentioned before, the overall goal of the UAV is to take off at its home base, consecutively fly to the interesting locations and take pictures, to finally return and land back at its home base. The current position (location and height) of the UAV, along with the status of taken pictures and distances to objects, form the relevant input for the policy. In IDP, the instructions that form the policy are represented using a rule-like syntax. As the policy contains dozens of such rules $^{5}$, only one, arbitrarily selected rule is denoted here for illustrative purposes. IF the UAV has sufficient power AND not all pictures are taken yet AND the current location does not have to be inspected (anymore) AND the UAV is at fly height AND the UAV is close to Restricted Area THEN the UAV will (try to) move away from the Restricted Area. In IDP syntax, this rule is denoted as:

$$
\begin{aligned}
& \forall t[\text { Time }]: \text { Plan }(t)=\text { MoveAwayFromRA } \\
& \leftarrow \text { AtNormalPower }(t) \wedge \neg \text { AllPicturesTaken }(t) \\
& \quad \wedge \neg \text { AtInspectionToDo }(t) \wedge \text { AtFlyHeight }(t) \wedge \text { CloseToRA }(t) .
\end{aligned}
$$

\footnotetext{
5 The policy of the application at hand is available at https://github.com/ VermaelenJan/Model-Checking-Drones-with-IDP/blob/master/Policy/Policy.pdf.
} 
Exactly one action must be returned for each possible state of the UAV. We say the policy should be complete. This can be verified exhaustively ${ }^{6}$ and is enforced by IDP as well. The policy at hand is found to be complete.

\subsection{Modeling the Time}

We denote two different ways of modeling Time by means of what the successor function Next(Time) looks like.

Finite Time. A classical time definition can be modeled using a partial successor function. Each time step is mapped to the next one $(0 \rightarrow 1,1 \rightarrow 2, \ldots, k-1 \rightarrow k)$. The final time step $(\mathrm{k})$ has no successor. In IDP syntax, Next(Time) is modeled as:

$$
\forall t[\text { Time }]: N \operatorname{ext}(t)=t+1 \leftarrow \operatorname{Time}(t+1) .
$$

Back Loop Time. A total time definition can be achieved by letting the final time step $k$ loop back to any previous one $l[3,4]$. IDP's knowledge base nature allows it to look for solutions in which states of the system are repeated. Infinite sequences of time steps can be considered, as long as they can be expressed using a $(\mathrm{k}, \mathrm{l})$ back loop. The model is extended with:

$$
\text { Next }(\text { MAX }[: \text { Time }])=\text { BackLoopTime. }
$$

where the value for BackLoop Time, representing $l$, can be chosen by IDP nondeterministically.

\subsection{Modeling the Properties}

Within IDP, model checking is achieved by BMC. To check property $f$, one adds the statement not $f$ to the base system and queries IDP to determine whether the new, extended theory is satisfiable. If the theory is satisfiable, a counterexample is found in which the negation of the property holds. The property is violated and hence does not hold in general. Some properties of the autopilot that are to be verified are the following:

1. The UAV does not fly into restricted areas.

2. The UAV achieves its inspection flight goal.

3. The UAV reaches back home, before running out of battery power.

\footnotetext{
${ }^{6}$ For the policy of the application at hand, proof of completeness is provided at https://github.com/VermaelenJan/Model-Checking-Drones-with-IDP/blob/mast er/Policy/Completeness\%20Policy/policy\%20complete.pdf.
} 
Since we need to look for scenarios in which the negation of the property occurs, we extend the theory as follows.

$$
\exists t[\text { Time }]: \text { Curr_DistanceToRA }(t)=0 .
$$

forces IDP to look for a scenario in which at some time step $t$, the UAV does reach the restricted area.

$$
\neg \exists t[\text { Time }]: \text { AllPicturesTaken }(t) \wedge \text { AtGroundHeight }(t) \wedge \text { AtHome }(t) .
$$

forces IDP to look for a scenario in which the UAV never reaches its goal.

$$
\neg(\text { AtHome }(M A X[: \text { Time }]) \wedge \text { AtGroundHeight(Max }[: \text { Time }])) .
$$

forces IDP to look for a scenario in which the UAV never returns home.

The properties can be divided into two groups: safety properties (1) and liveness properties (2 and 3 ). For safety properties, it is sufficient to find a single time step at which the property is violated. For liveness properties, on the other hand, we need to find an example of a complete flight trace in which the liveness goal does not get achieved.

Rather than applying a complete BMC iteration with an increasingly large bound, we opt for a single check with a fixed, well-chosen bound. In IDP, this corresponds to the use of a fixed time domain. In every flight scenario, after the UAV inevitably runs out of battery power, it will end up on the ground and remain there forever. In this final state, a back loop, as discussed in Sect. 3.4, occurs. If a safety property is not violated until this looping state is encountered, the property will not be violated anywhere in the entire execution trace. This statement is concordant with the observation by Biere et al. [4]. Furthermore, the infinite loop can be used to find counterexamples for liveness properties as well. Such a counterexample provides an infinite execution trace in which the liveness goal never gets achieved. Making use of a back loop, we avoid the main drawback of BMC, that is, the need for an endlessly increasing bound. We essentially only apply one BMC step with a sufficiently large bound.

Property 1 can be guaranteed, as no counterexample was found by IDP. Property 2 , related to reaching the goal, does not always hold. Depending on the initial battery level and the distances to be covered during flight, counterexamples can be found. Property 3, related to reaching back home, holds under some assumptions. For example, the number of non-deterministically failed actions has to be limited. If the policy takes this limit into account, the estimation it makes, regarding the power needed to return home, will always be sufficient to make sure the UAV can reach back home.

To improve the performance of the verification task, we look into reducing the time domain. We apply an approach related to Invariant Checking, as described by Biere et al. [2]. Only two consecutive time steps are taken into account simultaneously. This approach contrasts with a BMC approach, for which a complete flight scenario has to be considered. Biere et al. [3] state that the three steps in a proof with an inductive invariant are: the base case, the induction step, and 
the strengthening step. For the base case and induction step, we rely on IDP. As we use the property itself as the inductive invariant, the strengthening step holds trivially. For the base case, we have to check whether the property initially holds. This check is straightforward using a knowledge base tool such as IDP. By adding this requirement to the theory, IDP only looks for models in which the property holds in the initial time step. For the induction step, we apply a simplified BMC approach. We query IDP, looking for a counterexample. If the property holds at the initial time step (base case) and a time succession can be found after which the property is violated, the invariant, and thus the property, does not hold.

\section{Discussion}

The properties stated in Sect.3.5 are successfully verified for different concrete initializations of the world. If desired, the properties can easily be checked for different autopilot policies as well. We are able to check for each property whether or not it can get violated, and if so, a counterexample is provided by IDP.

\section{Discretization}

Physical domains, such as distance, can easily be discretized. For the application investigated in this research, a discretized approach is adequate. The use of discrete domains is sufficiently concrete, as the level of accuracy depends on the chosen unit (for example, express distance in centimeters instead of meters). Since an arbitrarily high finite precision can be achieved, we do not experience the need for continuous domains.

\section{Back Loop Time}

When modeling a realistic UAV, a back loop time structure might seem useless as the battery level decreases at each time step. However, a useful trick arises. Once the battery gets completely drained, a back loop allows us to express infinitely long paths. We can verify the properties using a fixed bound since the need for an endlessly increasing bound is avoided. For example, the bound can be set to a value greater than or equal to the initial battery level divided by the smallest possible power usage per time step.

\section{Performance}

As the intention is to execute representative verifications, realistic domain sizes would be desired. However, experience shows that the use of large domains in IDP is disastrous for its performance. As we verify properties imitating a single BMC step, we do not experience any advantages regarding the state explosion problem [6]. The use of smaller domains does not have to compromise the desired principle of concrete verification. An acceptable measure is to reduce the domain sizes but keep them as concrete. The properties will be checked on down-scaled worlds. For the system and properties at hand, it is plausible to assume that if 
no violations are found for such worlds, violations will not occur in realistically sized worlds either. This assumption is not valid if the properties depend on large values regarding certain domains.

Furthermore, the (correct) use of types is advantageous for the performance of IDP. Two important types for which additional measures regarding the domain size have been taken are the Height level of the UAV and the UAV's PowerUsage at each time step. In both cases, the use of subdomains succeeds to reduce the bulk of computations within IDP, yielding a performance gain.

Finally, the reduction of time steps by using Invariant Checking is also beneficial for the performance of the verification task. However, there are two major drawbacks. Firstly, only counterexamples with the chosen initialization state can be found. The check should be executed for every state in which violations might occur. Secondly, and perhaps more importantly, it yields that this way of checking properties is only applicable to safety properties, as a result of reducing the time domain. It is impossible to draw conclusions regarding any property of which the violation requires more than one time step succession. To remedy the first drawback, the initialization of the first time step is handed off to IDP to find as many violations as possible. The initial state of the system is set nondeterministically. All possible initializations are checked. For this purpose, the use of a knowledge base system is rather convenient. Furthermore, not only the initial state of the UAV but, for example, also the initialization of the distances that define the world can be taken care of by IDP. As a result, an even more complete search can be achieved. Since porting the model from BMC to IC (within IDP) requires no changes, IC only enhances the verification capabilities.

\section{Conclusion}

We outlined our model for the verification of a safety-critical UAV. Taking into account the assumptions and drawbacks we encountered, IDP can be used as a verification tool. The straightforward and compact way of modeling makes IDP suitable for a high-level approach of verification and facilitates acquiring quick proofs of concept. The counterexamples provided by IDP form excellent guidance for debugging purposes regarding the system at hand.

Using discrete domains, any arbitrary precision can be achieved. However, precise models require large domains, which are disastrous for the performance of IDP. Therefore, verification on down-scaled worlds is preferred. The introduction of a back loop, when possible, allows taking into account infinitely long execution paths. The need for an endlessly increasing bound during BMC is avoided. The use of subtypes yields to be advantageous for both the performance of IDP and the readability of the model. Furthermore, the application of Invariant Checking is also beneficial for the performance of the verification task. However, since Invariant Checking only takes into account two consecutive time steps, it is only applicable for safety properties.

Future work should focus on more realistic modeling of the system. The effects of executed actions and external influences are never precisely known. As mentioned at the end of Sect.2, probabilistic models are suitable to take 
into account more realistic effects. To obtain correct claims from such a model, however, the model itself first has to be guaranteed to be correct.

Acknowledgments. This research is partially funded by the Research Fund KU Leuven.

\section{References}

1. Alhawi, O.M., Mustafa, M.A., Cordeiro, L.C.: Finding security vulnerabilities in unmanned aerial vehicles using software verification. CoRR abs/1906.11488 (2019). http://arxiv.org/abs/1906.11488

2. Biere, A., Cimatti, A., Clarke, E.M., Fujita, M., Zhu, Y.: Symbolic model checking using SAT procedures instead of BDDs. In: Proceedings 1999 Design Automation Conference (Cat. No. 99CH36361), pp. 317-320, June 1999. https://doi.org/10. 1109/DAC.1999.781333

3. Biere, A., Cimatti, A., Clarke, E.M., Strichman, O., Zhu, Y.: Bounded model checking. In: Advances in Computers, vol. 58, pp. 117-148. Elsevier (2003). https:// doi.org/10.1016/S0065-2458(03)58003-2

4. Biere, A., Cimatti, A., Clarke, E., Zhu, Y.: Symbolic model checking without BDDs. In: Cleaveland, W.R. (ed.) TACAS 1999. LNCS, vol. 1579, pp. 193-207. Springer, Heidelberg (1999). https://doi.org/10.1007/3-540-49059-0_14

5. Bruynooghe, M., et al.: Predicate logic as a modeling language: modeling and solving some machine learning and data mining problems with IDP3. Theory Pract. Logic Program. 15(6), 783-817 (2015). https://doi.org/10.1017/ S147106841400009X

6. Clarke, E.M., Klieber, W., Nováček, M., Zuliani, P.: Model checking and the state explosion problem. In: Meyer, B., Nordio, M. (eds.) LASER 2011. LNCS, vol. 7682, pp. 1-30. Springer, Heidelberg (2012). https://doi.org/10.1007/978-3-64235746-6_1

7. Dinh, H.T., Cruz Torres, M.H., Holvoet, T.: Combining planning and model checking to get guarantees on the behavior of safety-critical UAV systems. In: ICAPS Workshop on Planning and Robotics (2018). https://lirias.kuleuven.be/retrieve/ 509824

8. Hoffmann, R., Ireland, M., Miller, A., Norman, G., Veres, S.: Autonomous agent behaviour modelled in PRISM - a case study. In: Bošnački, D., Wijs, A. (eds.) SPIN 2016. LNCS, vol. 9641, pp. 104-110. Springer, Cham (2016). https://doi. org/10.1007/978-3-319-32582-8_7

9. Kwiatkowska, M., Norman, G., Parker, D.: PRISM: probabilistic symbolic model checker. In: Field, T., Harrison, P.G., Bradley, J., Harder, U. (eds.) TOOLS 2002. LNCS, vol. 2324, pp. 200-204. Springer, Heidelberg (2002). https://doi.org/10. 1007/3-540-46029-2_13

10. Schumann, J., Visser, W.: Autonomy software: V\& V challenges and characteristics. In: 2006 IEEE Aerospace Conference, pp. 1-6. IEEE (2006)

11. Sirigineedi, G., Tsourdos, A., White, B.A., Żbikowski, R.: Kripke modelling and verification of temporal specifications of a multiple UAV system. Ann. Math. Artif. Intell. 63(1), 31-52 (2011). https://doi.org/10.1007/s10472-011-9270-x

12. Torens, C., Adolf, F.M., Goormann, L.: Certification and software verification considerations for autonomous unmanned aircraft. J. Aerosp. Inf. Syst. 11(10), 649664 (2014) 